\title{
Dynamical response of the magnetotail to changes of the solar wind direction: an MHD modeling perspective
}

\author{
V. A. Sergeev ${ }^{1}$, N. A. Tsyganenko ${ }^{1}$, and V. Angelopoulos ${ }^{2}$ \\ ${ }^{1}$ Institute of Physics, St. Petersburg State University, St. Petersburg, Russia \\ ${ }^{2}$ IGPP/UCLA, Los Angeles, USA
}

Received: 11 March 2008 - Revised: 17 June 2008 - Accepted: 10 July 2008 - Published: 6 August 2008

\begin{abstract}
We performed global MHD simulations to investigate the magnetotail response to the solar wind directional changes ( $V_{z}$-variations). These changes, although small, cause significant variations of the neutral sheet shape and location even in the near and middle tail regions. They display a complicated temporal response, in which $\sim 60$ to $80 \%$ of the final shift of the neutral sheet in $Z$ direction occurs within first 10-15 min (less for faster solar wind), whereas a much longer time (exceeding half hour) is required to reach a new equilibrium. The asymptotic equilibrium shape of the simulated neutral sheet is consistent with predictions of Tsyganenko-Fairfield (2004) empirical model. To visualize a physical origin of the north-south tail motion we compared the values of the total pressure in the northern and southern tail lobes and found a considerable difference (10-15\% for only $6^{\circ}$ change of the solar wind direction used in the simulation). That difference builds up during the passage of the solar wind directional discontinuity and is responsible for the vertical shift of the neutral sheet, although some pressure difference remains in the near tail even near the new equilibrium. Surprisingly, at a given tailward distance, the response was found to be first initiated in the tail center (the "leader effect"), rather than near the flanks, which can be explained by the wave propagation in the tail, and which may have interesting implications for the substorm triggering studies. The present results have serious implications for the data-based modeling, as they place constraints on the accuracy of tail magnetic configurations to be derived for specific events using data of multi-spacecraft missions, e.g. such as THEMIS.
\end{abstract}

Keywords. Magnetospheric physics (Magnetospheric configuration and dynamics; Magnetotail; Solar windmagnetosphere interactions)

Correspondence to: V. A. Sergeev

(victor@geo.phys.spbu.ru)

\section{Introduction}

The magnetic tail of the magnetosphere is aligned parallel to the solar wind (SW) flow, which is nearly radial. Accordingly, a suitable coordinate system is the Geocentric Solar Magnetospheric (GSM), in which the $\mathrm{X}$-axis is oriented along the Earth-Sun line and, hence, antiparallel to the average SW flow. The GSM coordinates are widely used as a basic coordinate system for the magnetospheric modeling and in various studies of the Earth's magnetotail processes. In fact, the solar wind deviates from the radial direction, although this deviation is not large. First of all, there is a $\sim 4^{\circ}$ aberration due to Earth's orbital motion. Superimposed on that systematic effect, there are irregular variations of the solar wind direction. As shown in a statistical study of 5-min average velocity vectors (Tsyganenko and Fairfield, 2004; referred henceforth as TF04), the most probable angular deviation is about $2.5^{\circ}$, with only $5 \%$ of vectors deviated beyond $7^{\circ}$. However, at sufficiently large tailward distances even the relatively small deviations may have considerable effect on the position of the tail neutral sheet (NS). For example, already at the distance of Cluster apogee $\left(18 R_{E}\right)$ the windsock effect due to $\sim 7^{\circ}$ deviation of the SW direction can produce, roughly, a $\sim 2 R_{E}$ shift of the NS with respect to the spacecraft, on the order of the thickness of the current sheet. This effect dramatically increases in the distant tail, where it was first recognized and investigated on the basis of data from the ISEE-3 deep tail orbits. Hones et al. (1986) suggested to adjust the GSM coordinate system by redirecting the $\mathrm{X}$-axis antiparallel to the currently observed solar wind flow vector, rather than along the Earth-Sun line, and use thus defined GSW system for representing more accurately the magnetotail configuration. That system was later used for organizing magnetometer data in the modeling studies of the tilt-dependent shape of the tail NS (Tsyganenko et al., 1998; TF04). Unfortunately, a number of effects may limit the accuracy of those models: the solar wind $V_{y}$ and $V_{z}$

Published by Copernicus Publications on behalf of the European Geosciences Union. 


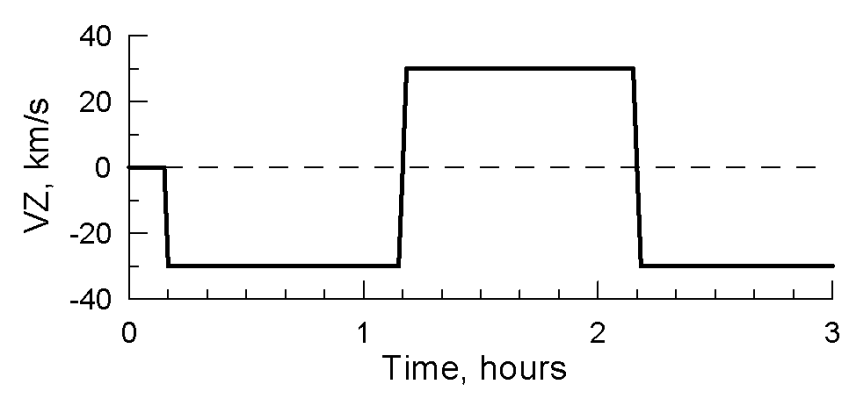

Fig. 1. Variations of $V_{z}$-component of solar wind velocity at the frontside boundary of the simulation domain $\left(X_{f}=33 R_{E}\right)$.

components may differ at different locations in the inhomogeneous solar wind, the fronts of particular inhomogeneities may be significantly tilted with respect to $Y Z$ plane, instrumental offsets may also be a problem, etc.

The principal factor providing the largest contribution to the chaotic windsock deflections of the tail is the temporal variability of $V_{z}$ and $V_{y}$ flow components. Surprisingly, many important details of the magnetotail response to abrupt changes of the solar wind direction are not yet known, in particular, how quickly its new equilibrium is established. Even after decades of spacecraft observations we still know very little about the dynamical characteristics of the windsock effect, due to difficulties in resolving them using data of only a few spacecraft usually available at a time. That was one of conclusions of Kaymaz et al. (1995), who attempted to investigate a couple of events with concurrent observations in the solar wind and in the distant magnetotail. Those authors also pointed out that more than one factor might contribute to form the temporal response. In particular, besides the propagation of solar wind discontinuity (at which the SW direction changes) along the tail with typical SW velocities of 3-6 $R_{E} / \mathrm{min}$, the disturbance can propagate much faster inside and along the tail (Alfvén or fast sonic wave speed may be as large as $\sim 10-20 R_{E} / \mathrm{min}$ in the midtail lobe). Waves also propagate with different velocities in the tail and in the magnetosheath, which also contributes to the complexity of expected response.

In this paper we try to fill that gap by investigating the temporal response of the tail as deduced from a first-principle simulation. Since that response is expected to be an inherently MHD process, we use the advantage of MHD simulations, allowing us to model the tail windsock motion and to infer its global dynamical properties. In particular, we address the tail's response to isolated directional discontinuities in the SW flow, by concentrating on such a specific characteristic as the dynamic response of the tail NS, the central surface of the tail current sheet where the field $B_{x}$-component changes sign. This also allows us to compare the simulation results with the TF04 empirical model of the NS, to test how close to reality are the MHD results. Derivation of the timescales of the tail dynamic response is one of main goals of this work. Another basic characteristic studied here is the response of the tail pressure, the driving force of the transverse motions in the magnetotail.

\section{Simulation results}

We have run the simulations at the Community Coordinated Modeling Center (CCMC) facility, operating at NASA GSFC. Standard setups of two different global MHD codes supported at CCMC have been used, to make sure that the obtained results are code-independent. The first code, BATSR-US, solved the ideal MHD equations using numerical diffusion only (see Powell et al., 1999, for the details). The simulation domain was $[33,-351] R_{E}$ in $X$, and $\pm 48 R_{E}$ in both $Y$ and $Z$. The second one, OpenGGCM, solved the MHD equations with additional dissipation (see, e.g. Raeder, 2003). The simulation domain was $[24,-300] R_{E}$ in $X$, in comparison with BATS-R-US, the second code was found to yield higher activity in the magnetotail, with many more transient and localized mesoscale structures. In both codes we used standard spatial resolutions with the grid size $0.5 R_{E}$ in the equatorial region, near the magnetopause, and in the inner magnetosphere, and with a larger grid-size (up to $2 R_{E}$ ) in other regions. Homogeneous ionospheric conductivity of $5 \mathrm{MHO}$ has been applied, with no corotation. The dipole tilt was fixed to be zero in GSM coordinates.

All the solar wind and IMF parameters but $V_{z}$ were fixed throughout the simulations, with the purpose to isolate the effects of directional changes of the solar wind flow. In this paper, we primarily analyze runs "Victor_Sergeev_091907_1" (BATS-R-US, hereafter referred as \#5), and "Victor_Sergeev_100807_1" (OpenGGCM, hereafter referred as \#6), in which the IMF vector was set fixed at $[3 ; 0 ; 2] \mathrm{nT}$ and the solar wind parameters were fixed at $N=20 \mathrm{~cm}^{-3}$ (density), $T=6 \times 10^{4 \circ} \mathrm{K}, V_{x}=300 \mathrm{~km} / \mathrm{s}$ and $V_{y}=0 \mathrm{~km} / \mathrm{s}$ during both the initialization and simulation periods. The only variable parameter was $V_{z}$, initially set at $V_{z}=0$ (Fig. 1), and then changed in a stepwise fashion to $-30 \mathrm{~km} / \mathrm{s}$, then to $30 \mathrm{~km} / \mathrm{s}$, and then again to $-30 \mathrm{~km} / \mathrm{s}$. Each transition lasted $2 \mathrm{~min}$ and was followed by 58 -min interval of constant $V_{z}$. In this run, $\left|V_{z} / V_{x}\right|$ reached $1 / 10$, so that the solar wind flow deflected by $\sim 6^{\circ}$ from strictly antisolar direction. The above solar wind conditions have been set at the front side of the simulation box. The Earth's dipole was kept perpendicular to Sun-Earth line at all times, so that the tail NS initially (i.e. while $V_{z}=0$ ) coincided with the $Z=0$ plane. As $V_{z}$ becomes nonzero, the dipole tilt is no longer zero in the GSW coordinate system and, hence, the neutral sheet surface is no longer a plane. During the simulation period the signatures of magnetic reconnection were permanently observed in the midtail, with higher intensity and at closer geocentric distance of the flow reversal $\left(X \sim-15 \ldots-20 R_{E}\right)$ in the tail 
a) $\quad 01 / 01 / 2000$ Time $=00: 00 ; 00 \mathrm{y}=8.00 \mathrm{R}_{\mathrm{E}}$

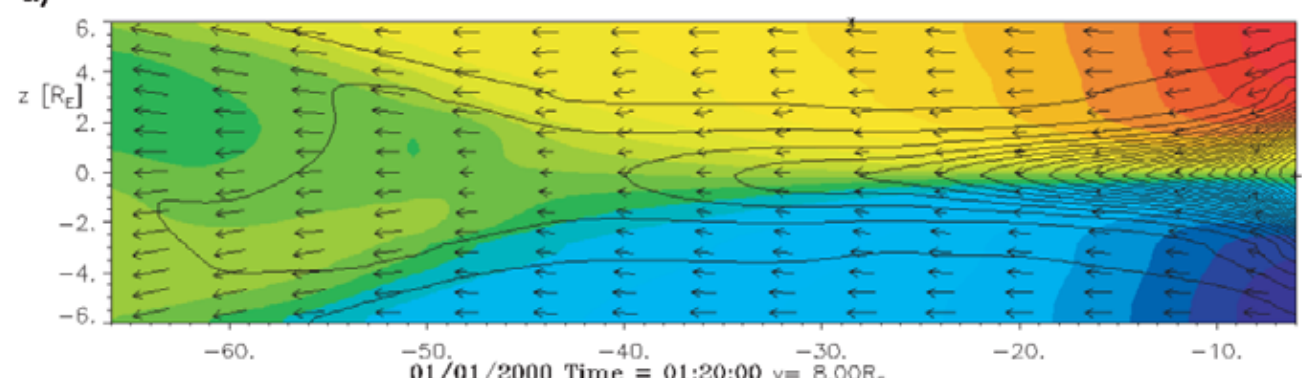

$\uparrow \vee\left[\frac{k m}{5}\right]$

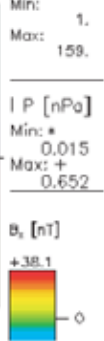

'b)

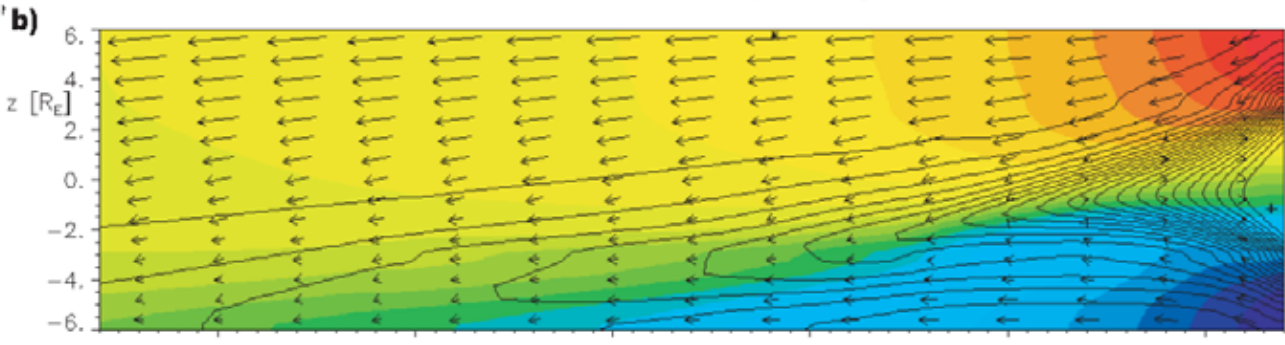

$\uparrow \vee\left[\frac{\mathrm{km}}{\mathrm{m}}\right]$

c)

01/01/2000 Time $=01: 38: 00 y=8.00 R_{\mathrm{E}}$

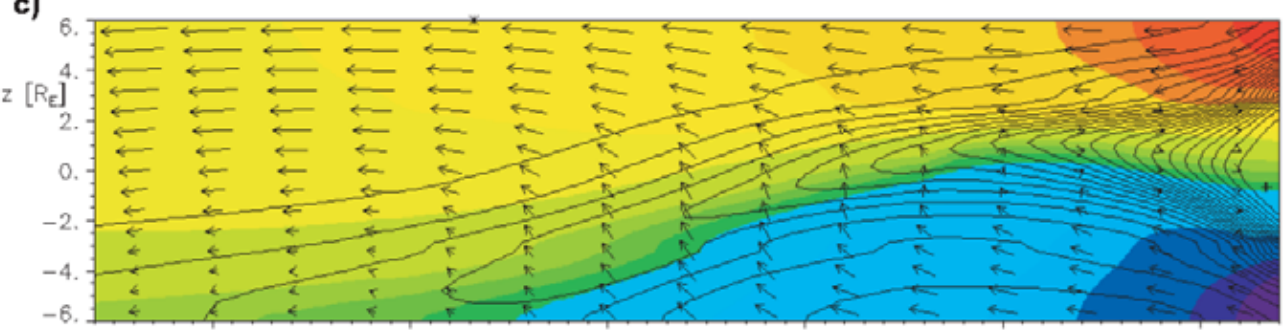

01/01/2000 Time $=02: 20: 00 y=8.00 R_{E}$

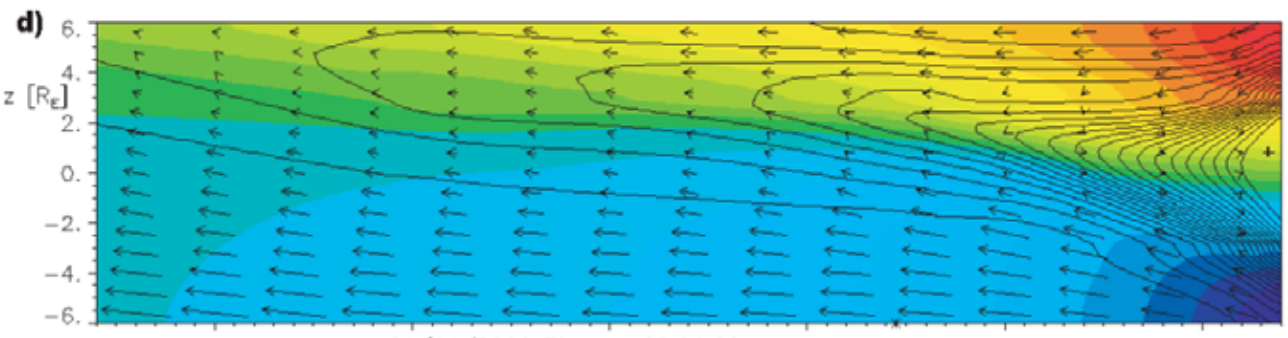

01/01/2000 Time $=02: 38: 00$ y= 8.00R

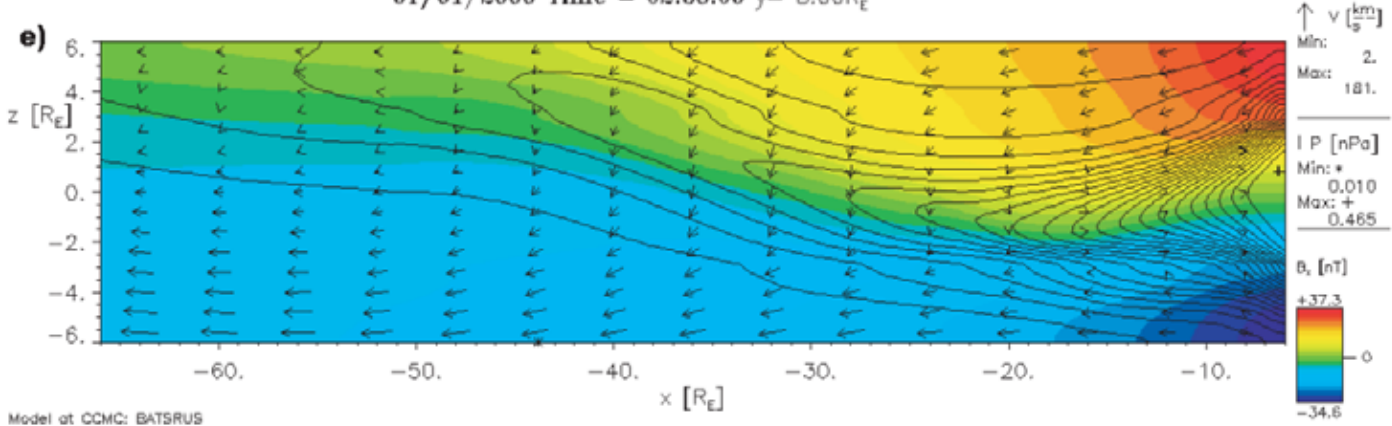

Fig. 2. Examples of flow velocity vectors, plasma pressure isointensity contours and magnetic $B_{x}$-component distribution (color-coded) in the meridional cross-section at $Y=8 R_{E}$ during the simulation run \#5 (BATS-R-US). The pressure isointensity contours illustrate the configuration of magnetic field lines (due to $P \sim$ const along those lines). 


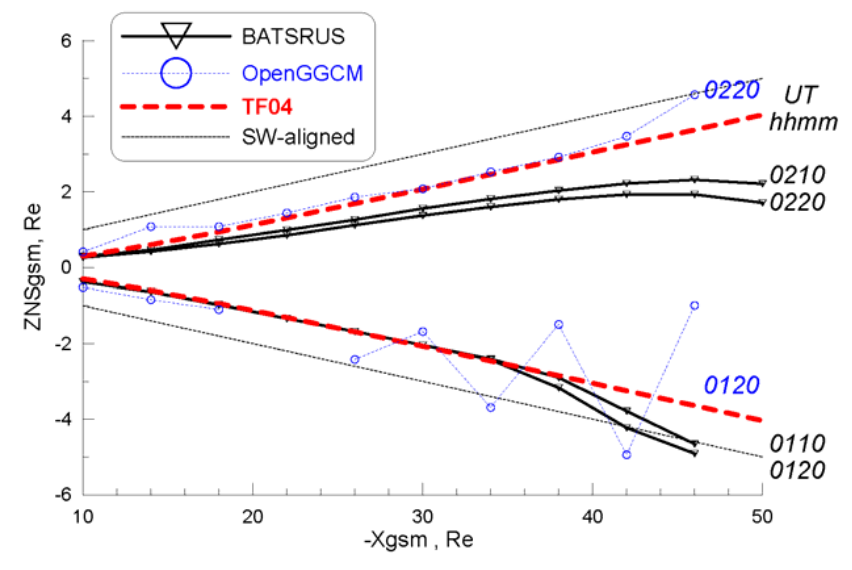

Fig. 3. NS positions in $X Z$ plane after $\sim 1$-h period of the solar wind flow with nonzero $V_{Z}$ components (near 01:20 UT and 02:20 UT, see Fig. 2). Results are shown from the runs \#5 (black, BATS-R-US) and \#6 (blue, OpenGGCM). Red dashed line shows predictions by the empirical model TF04, and the dotted line shows, for reference, the line through the Earth center, parallel to the asymptotic solar wind flow.

center than near flanks, throughout the entire simulation period.

Figure 2 illustrates the structural changes of the equatorial tail in response to the changing solar wind direction. Initially the NS lies in the $Z=0$ equatorial plane (a). As the SW discontinuity travels over the midtail (plates (c), (e)), the originally planar sheet gets deformed and becomes a curved surface, and this reorganization of the magnetotail is accompanied by a significant cross-tail plasma flow in the northsouth direction. At the end of $\sim 1$-h stable $V_{z}$ period (b, d), the NS appears to be nearly aligned with the SW flow, suggesting that the tail got quite close to the new equilibrium and the NS reached its new asymptotic position. That will also be seen below in the time plots of the NS locations in Figs. 5 and 6.

To infer the asymptotic shape of the NS (i.e. the $B_{x}$ reversal surface in the current sheet) at various $(X, Y)$ positions and to quantitatively investigate its dynamics, we used the profiles of the magnetic field component $B_{x}(Z)$ interpolated to an equidistant grid, with $0.2 R_{E}$ resolution in $Z$. To obtain a more accurate estimate of the $B_{x}$-reversal, we used 11 points of so obtained $B_{x}(Z)$ profile covering $2 R_{E}$ centered at the apparent $B_{x}$ reversal location in $Z$, we approximated $B_{x}(Z)$ by a linear regression fit. Besides having estimated the location ZNS of the NS from this fit, we also controlled the correlation coefficient of the fit, and in case if its value fell below 0.8 , the results were discarded as potentially uncertain. In this way we avoided the turbulent regions with uncertain NS location, e.g. those in the dynamical plasmoids, or in the regions between two colliding plasma streams, etc. This was especially important in Open GGCM simulation which displayed much more disturbed plasma patterns near the $B_{x}$-reversal as compared to the BATS-R-US simulation. (In the following, we will mostly illustrate results for the BATS-R-US run, also showing some results for the OpenGGCM to address the consistency between the two codes, as concerns the global features.)

We first display the asymptotic NS shape in the meridional $(X Z)$ plane (Fig. 3). It looks similar to predictions of the TF04 empirical model, which takes into account both the angular deviations of the solar wind flow and the non-zero dipole tilt that now appears in the new (GSW) coordinate system. Whereas the change of the solar wind direction provides the main contribution in the $X Z$ plane, the effects of the dipole tilt are easier to recognize in the $Y Z$ plane, in which the neutral sheet also exhibits a tilt-related warping, extensively studied both theoretically and empirically (Tsyganenko, 1998; TF04, and references therein).

Comparison of predicted (TF04) and simulated asymptotic NS shapes in the $Y Z$ plane shown in Fig. 4 clearly indicates the warping of the NS surface. The agreement observed in both cross-sections (at $X=-14 R_{E}$ and $-30 R_{E}$ ) in the runs, with both codes yielding similar results, is an encouraging fact showing that global MHD simulations provide an accurate description of the large-scale tail deformations. The smooth warping shape is more robustly reproduced by both codes in the near-Earth region $\left(X=-14 R_{E}\right)$ where it nicely agrees with the empirical NS surface. At farther downtail distance $\left(-30 R_{E}\right)$, the gross features of the warping are still reproduced fairly well, but the simulated neutral sheet is more structured and unstable. It is also of note that the simulated NS shapes at positive/negative tilts are not identical, especially near the flanks (this is possibly an effect of the IMF $B_{x}$ component, to be addressed in a future separate study).

To analyze the dynamical response, we plotted in Fig. 5 time variations of the neutral sheet position $\left(Z_{\mathrm{NS}}\right)$ in three planes parallel to the $X Z$ plane (at $Y=0,8$, and 14), and at four locations on the $\mathrm{X}$-axis, to investigate the dynamics along the tail. To facilitate the timing and comparison with the passage of the SW discontinuity over those locations, we also plotted for each distance the corresponding stepwise reference profiles, obtained by propagating the discontinuity from the frontside simulation boundary $\left(X_{f}=33\right)$ to the distance of interest, adding the time shift $\left.d T=\left(X_{f}-X\right) / V_{x}\right)$. Using thus obtained local solar wind flow direction, we then calculated the ZNS location from the TF04 model at the intermediate distance $Y=8$ from the midnight plane. That procedure provided a reference profile (thin black rectilinear trace in Fig. 5), corresponding to a kind of instantaneous tail response to the passage of SW directional discontinuity, which facilitated the timing of the discontinuity passage and also gave a reference for the amplitude of expected neutral sheet shift, to compare it with the simulation results.

The first obvious result of Fig. 5 is that the major part of neutral sheet reconfiguration is controlled by the propagation of the solar wind discontinuities. Also, the agreement 


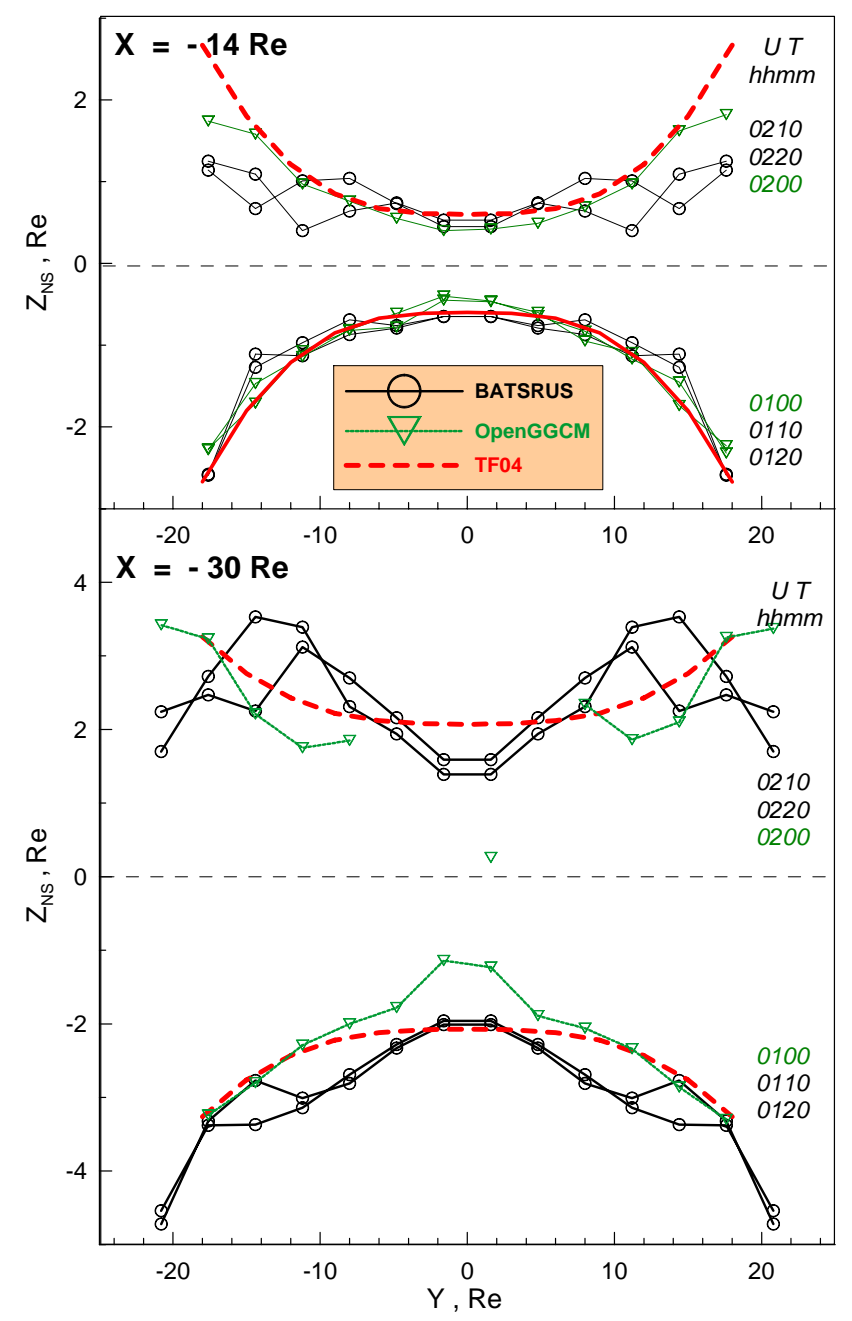

Fig. 4. The same as in Fig. 3 but for the asymptotic neutral sheet positions in $Y Z$ plane at two tail cross-sections $\left(X=-14 R_{E}\right.$ and $\left.-30 R_{E}\right)$.

between the observed and predicted neutral sheet shifts at distances ranging from the near tail $\left(10 R_{E}\right)$ to the midtail (up to $42 R_{E}$ ) is another proof of the consistency between the simulated and real deformations of the NS. However, the dynamics of the simulated response is more complex than according to the idealized instantaneous model with the rectilinear response profile. One may clearly see the signatures of the bimodal response pattern. The main change (from $60 \%$ to $80 \%$ of the asymptotic value, depending on the distance) occurs during the first $10-15 \mathrm{~min}$ after the passage of the discontinuity. It is then followed by a more gradual change, which looks a little different at different $Y$ cross-sections. In particular, an interesting detail is the signature of a longperiod oscillation in the near-flank tail region. Also, it appears that during the intervals of constant nonzero $V_{z}$ as long as $1 \mathrm{~h}$ in our runs, the system does not reach yet the final

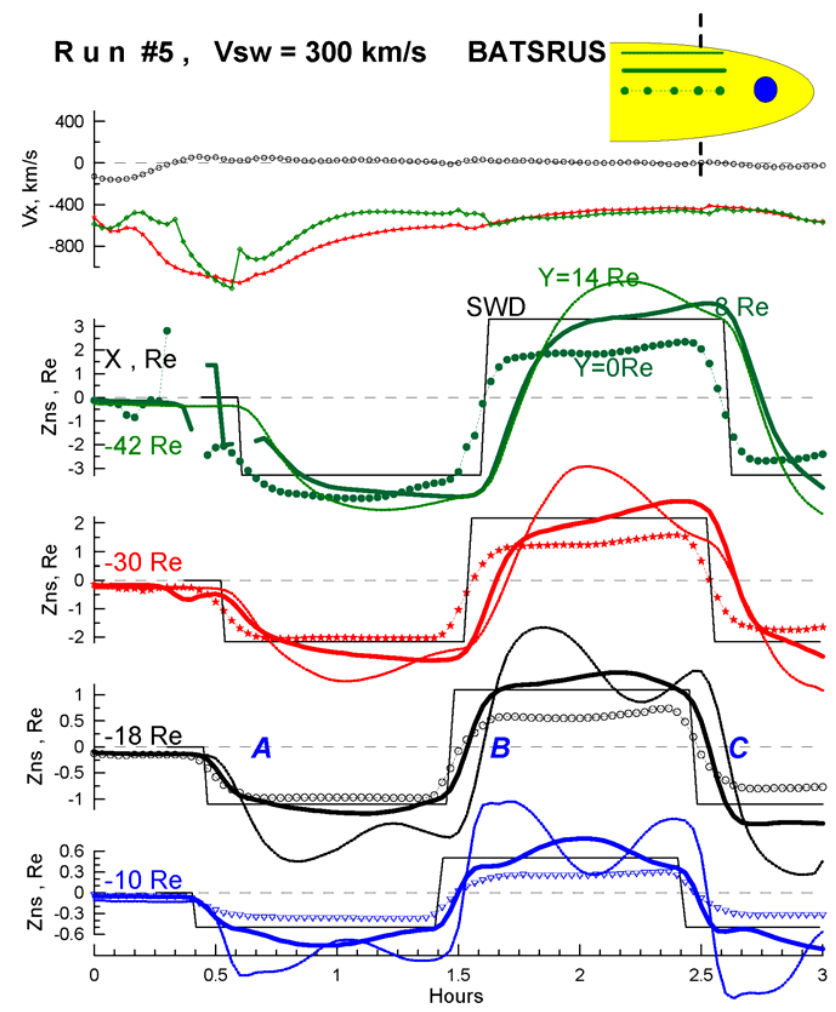

Fig. 5. Time variations of the NS location at different $X$ and $Y$ in the BATS-R-US simulation. The circular dots, thick and thin lines show the simulated shifts at $Y=0,8$ and $14 R_{E}$, respectively, see, for reference, a sketch in the right upper corner. (A few gaps correspond to cases when the NS position could not be accurately determined). The black rectilinear trace at each $X$ shows the hypothetical reference profile that would result from the instantaneous response of the NS to the passage of the directional discontinuity, with the amplitude given by the TF04 empirical model. The plasma flow velocity in the neutral sheet at $Y=0$ is given for reference.

equilibrium state. This result should be studied in more detail in future simulations. One more interesting and unexpected result is that, at a given tail cross-section, the changes start first near the tail center. This effect is small in the near tail $\left(X>-15 R_{E}\right)$, but becomes significant at 30 and $42 R_{E}$ in Fig. 6, where the time difference between the variation onsets at $Y=0$ and $Y=8 R_{E}$ is already as large as 6-8 min. We will further discuss that feature in the next section.

The driving force that causes the tail reconfiguration is the buildup of the pressure difference across the tail that results from the opposite changes of the SW dynamic pressure exerted on its northern and southern lobes. The north-south pressure difference drives the large-scale vertical plasma flow component that is seen in the simulation results (Fig. 2c, d). To visualize how the pressure difference builds up and works, we calculated the total (magnetic plus plasma) pressure at $Z=+6 R_{E}\left(\mathrm{PT}^{+}\right)$and $Z=-6 R_{E}$ 
$\left(\mathrm{PT}^{-}\right)$at some $(X, Y)$ location, and then calculated the normalized north-south pressure asymmetry parameter as $\mathrm{AP}=\left(\mathrm{PT}^{+}-\mathrm{PT}^{-}\right) /<\mathrm{PT}>$. Fig. 6 demonstrates the expected consistency in the behavior of AP and ZNS at any location near the neutral sheet. One clearly sees the buildup of pressure difference up to $\sim 10-15 \%$ in the AP parameter, associated with the vertical motion of the NS, with a very similar behavior in both simulation runs. However, the temporal variation of the pressure difference changes with the distance. At large tailward distances (e.g. at $42 R_{E}$ in Fig. 6) this difference fades after $\sim 15 \mathrm{~min}$, whereas in the near tail (e.g. at $18 R_{E}$ in Fig. 6) the pressure difference stays nearly at the $\sim 10 \%$ level for about an hour, until the next change of the solar wind flow direction, initiating the next reconfiguration.

\section{Discussion}

We performed simulation runs, in which the only changing external parameter, the north-south component of the solar wind flow velocity, was found to have important consequences for the tail configuration and dynamics, providing several important practical lessons. First, the consistency of the simulated asymptotic shape of the NS with the one derived empirically from spacecraft observations (Figs. 3, 4) demonstrates that MHD simulations provide realistic predictions and can serve as a valuable tool in the studies of largescale magnetospheric dynamics in the regions, where the MHD approximation remains valid. Although two different codes performed differently in details (with more mediumscale perturbations in the OpenGGCM code than in BATSR-US), their results (when possible to compare) were consistent in the prediction of the large-scale features, namely, the neutral sheet shape (Figs. 3, 4) and pressure asymmetry (Fig. 6). Second, the effects of relatively small directional changes (only $6^{\circ}$ angular deviation of the solar wind) are shown to provide significant effects in the tail. For reference, at $x=-18 R_{E}$ (Cluster apogee) they include a $\sim 1 R_{E}$ vertical shift and 1-2 $R_{E}$ warping amplitude of the current sheet, as well as the $10-15 \%$ variations of the total pressure difference between the northern and southern lobes. Certainly, the tail (solar wind) orientation and its changes are among the basic parameters to be monitored when studying particular magnetic configurations in specific events, using THEMISand CONSTELLATION-class missions. A third lesson is that temporal response of the tail to the solar wind directional changes is rather complicated and takes a long time, so it is difficult to predict based on the static approach in case of time-varying SW conditions.

An interesting and unexpected result of simulations is the "leader effect". Both the pressure difference and the NS location start to change first in the tail center but not near the flank, and this definitely occurs well before the passage of the SW discontinuity over the corresponding distance (Fig. 6). This effect is small in the near tail $\left(X>-15 R_{E}\right)$, but be- comes significant at 30 and $42 R_{E}$ in Fig. 6, where the time difference between the variation onsets at $Y=0$ and $Y=8 R_{E}$ is already as large as 6-8 $\mathrm{min}$. In the discontinuity-related coordinate system moving at the solar wind speed of $300 \mathrm{~km} / \mathrm{s}$, this time difference gives the scale-size along the tail about $15-25 R_{E}$, which is comparable to the tail radius at these locations. A useful way to visualize that effect is to plot the distribution of $E_{y}$ in the $X Z$ plane, as shown in Fig. 7. Since $E_{y}=-[V \times B]_{y}$ in the ideal MHD, the main contribution in the lobes comes from the term $V_{z} B_{x}$, it therefore helps to visualize the regions where the large-scale vertical plasma flow component is excited in the lobes by the north-south pressure gradients, Fig. 7. (Note that $E_{y}$ has opposite signs in northern and southern lobes in the case of a vertical flow in the same direction). Red dashed lines in Fig. 7 sketch the leading smooth fronts of the lobe regions where the cross-tail vertical flows have been induced in response to the changing solar wind direction. It clearly shows that a disturbance in the tail center propagates ahead of (and together with) the SW discontinuity, which is also clearly seen in this kind of presentation. Explanation of these effects, as well as of the Ydependence seen in Figs. 5, 6 (effects at $Y=8$ and $Y=14 R_{E}$ lag behind those at $Y=0$ ) plausibly includes the Alfvén velocity dependence on $Z$ and $Y$. The smallest density and largest $V_{A}$ are known to occur (and are observed in the simulations) in the lobe portion adjacent to the plasma sheet in the tail center. A part of the magnetopause behind the discontinuity, where the $V_{z}$ and dynamic pressure changed, acts as a source of perturbations. With the Alfvén travel time from tail boundary to the tail center about 2-3 min, the wave propagated across B from the source may launch the transverse Alfvén wave propagated tailward very fast. For reference, the maximal Alfvén velocity at $X=-15 R_{E}$ and $X=-25 R_{E}$, was $9.5 R_{E} / \mathrm{min}$ and $6 R_{E} / \mathrm{min}$ in the tail center, correspondingly. Fast propagation speeds in the tail lobes compared to the slow propagation of the SW discontinuity along the tail (treated as an external perturbation) mean also that the magnetotail quickly reaches a kind of equilibrium (quasi-static in the frame of discontinuity) for this perturbation. Therefore the leader effect can also be discussed from a different perspective of static models. Although the perturbation geometry is very different, our case has some analogy with Collier et al. (1998) model of the sudden SW pressure jumps. Their static solution also has a property that in the tail center the perturbation starts by $\Delta X \sim 1 \mathrm{RT}$ (RT is a tail radius) ahead of the sharp discontinuity location in $X$ (see their Fig. 8). The discussed "leader" effect, to our knowledge, was not yet reported before. We expect it may have important implications for studies of magnetotail effects of the solar wind discontinuities, e.g. when studying the substorm triggering problem. Kaymaz et al. (1995), who investigated a couple of events with clear SW directional changes with concurrent observations by two spacecraft in the solar wind and in the distant magnetotail, were first to point out that different processes may contribute to the formation of 


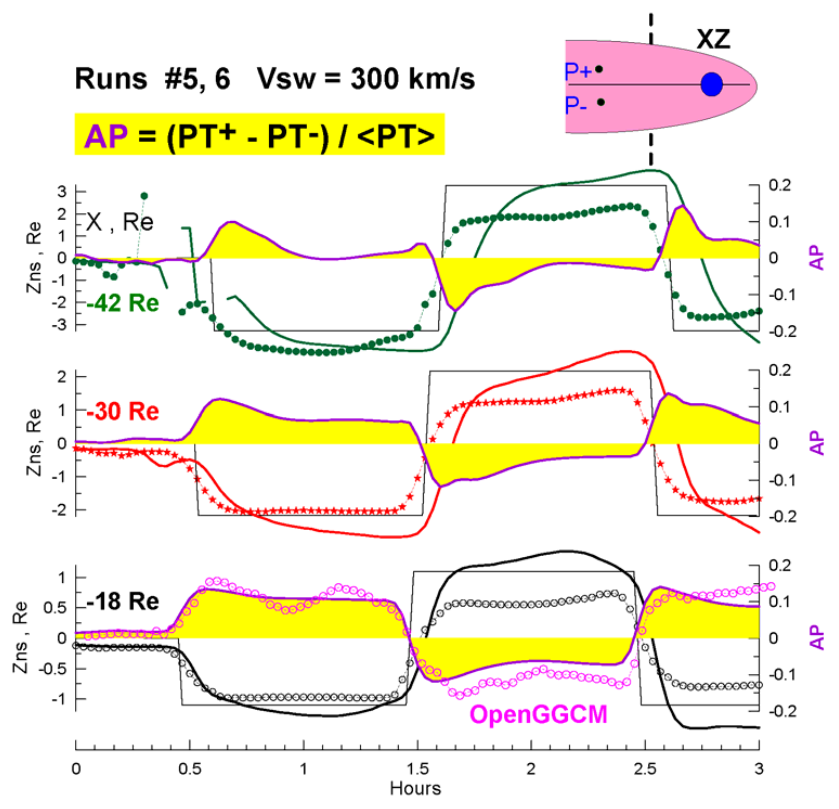

Fig. 6. Neutral sheet location at $Y=0$ and $8 R_{E}$ (circle dots and solid lines, respectively) and a normalized pressure anisotropy AP (at $Y=0)$ at different tailward distances, according to BATS-R-US simulation. The pressure asymmetry variation at $X=-18 R_{E}, Y=0 R_{E}$ in the OpenGGCM simulation is also shown for comparison (circular dots, magenta).

the tails dynamical response. They noticed the importance of the SW discontinuity propagation, of fast wave propagation along the magnetotail (with fast velocities in the lobes) and the wave propagation along the magnetosheath. Our simulation study clearly demonstrates the importance of, at least, the first two effects. Kaymaz et al. (1995) also discussed a few possible extreme idealized models of the magnetotail response to the SW directional changes. The first one was a "windsock" model, with nearly massless magnetotail which quickly aligns with the changing solar wind direction. The second one was the "driftwood" model, with a "heavy" tail, which is slowly pushed by the lateral force that develops due to the pressure difference on different sides of the magnetotail, it was not supported by the data presented by Kaymaz et al. (1995). The simulated dynamical response in our cases indicates the presence of both types of behavior, with a quick (10-15 min) initial tail reaction (in analogy to the windsock behavior) and a slower relaxation to a new equilibrium (as expected in the driftwood-like behavior).

\section{Conclusions}

Global MHD simulations appear as an effective tool to investigate the large-scale response of the magnetotail to the solar wind directional $\left(V_{z}\right)$ changes. These changes, although small, cause significant pressure asymmetry and drive the

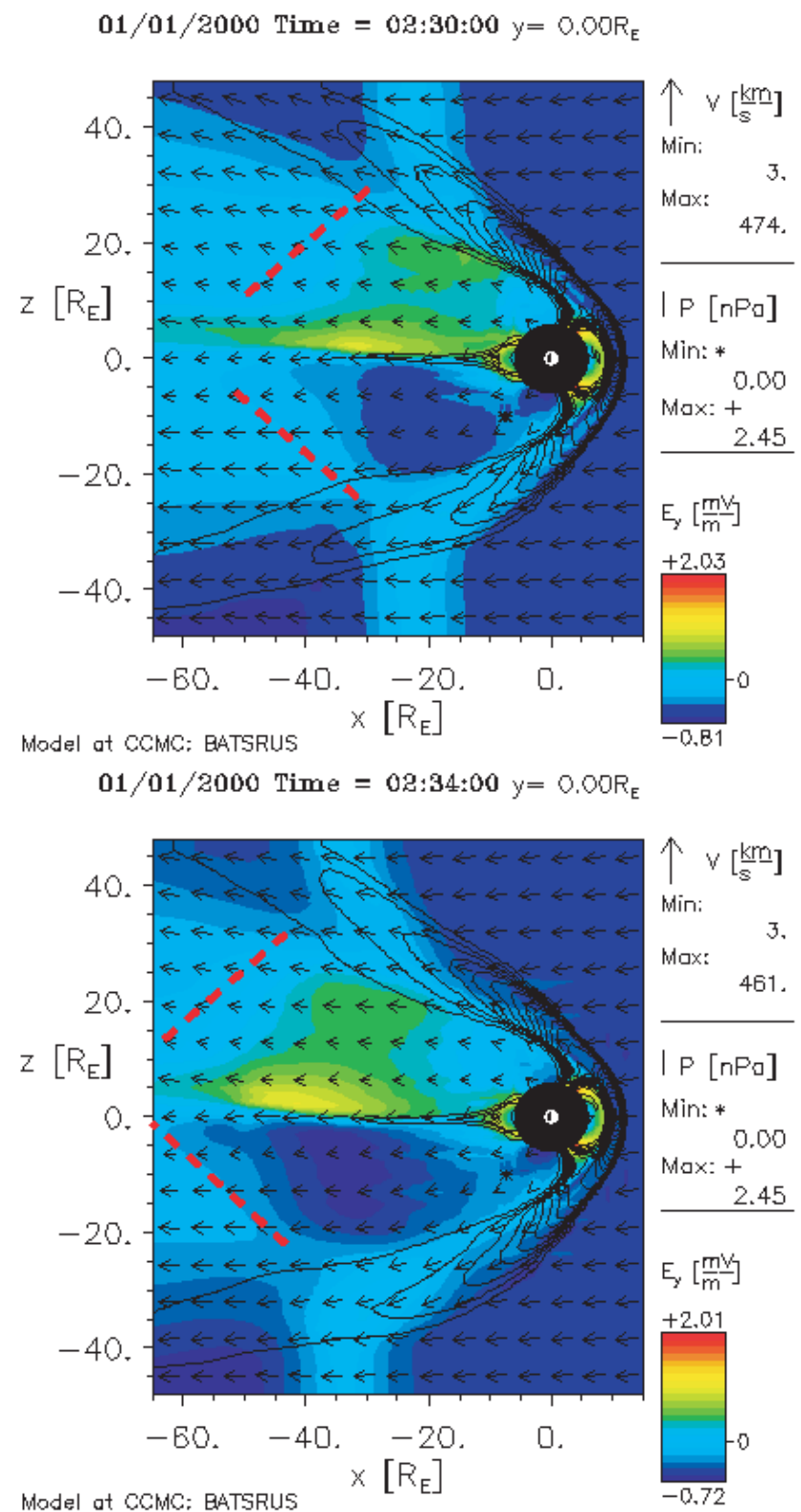

Fig. 7. Distribution of $E y=-[V \times B]_{y}$ in the $X Z$ plane plotted together with pressure isointensity contours (to visualize the magnetosheath) and the flow vectors during the passage of SW directional discontinuity over the tail. Red dashed lines sketch the geometry of smooth leading fronts of the lobe regions where the cross-tail vertical flows have been induced in response to the changing solar wind direction.

cross-tail plasma flows that induce considerable variations of the NS shape and location, even in the near and middle tail regions. The temporal response appears to be rather complicated, with a 10-15 min timescale (shorter for faster solar wind) for the most part of the change, covering roughly from 60 to $80 \%$ of the final shift of the NS, whereas a much longer 
time (exceeding $0.5 \mathrm{~h}$ ) is required to approach a new equilibrium. We found that asymptotic shape of the simulated NS near the equilibrium is consistent with predictions of the empirical model TF04. To visualize a physical origin of the north-south tail motion, we compared the total pressures in the northern and southern tail lobes and found their considerable difference (as large as $10-15 \%$, for only $6^{\circ}$ change in the solar wind direction). That pressure difference is initiated during the passage of the discontinuity, and it is responsible for the vertical shift of the NS, although some remnant pressure difference remains in the near tail near equilibrium, as a result of current sheet warping. An interesting "leader" effect of the NS response, starting first in the tail center rather than near its flanks (at the same distance in the tail) was observed and explained by the fast propagation of perturbation along the low-density tail lobes in the tail center. The results have implications and put constraints on data-based modeling efforts, aimed at obtaining accurate magnetotail configurations in specific events using multiple-spacecraft missions, e.g. THEMIS.

Acknowledgements. We thank the developers of SWMF/BATS-RUS and OpenGGCM global MHD simulation runs, which have been performed at CCMC through their public Runs on Request system (http://ccmc.gsfc.nasa.gov). The CCMC is a multi-agency partnership between NASA, AFMC, AFOSR, AFRL, AFWA, NOAA, NSF and ONR. We thank Marianna Holeva for her help in the paper preparation. The work by V. A. Sergeev and N. A. Tsyganenko was supported by the RFBR grants 07-02-91703, 07-05-91109 and CRDF grant 2861.

Topical Editor I. A. Daglis thanks Z. Kaymaz and M. Palmroth for their help in evaluating this paper.

\section{References}

Collier, M. R., Slavin, J. A., Lepping, R. P., Ogilvie, K., Szabo, A., Laakso, H., and Taguchi, S.: Multispacecraft observations of sudden impulses in the magnetotail caused by solar wind pressure discontinuities: Wind and IMP8, J. Geophys. Res., 103, 17 293-17 305, 1998.

Hones, E. W., Zwickl, R. D., Fritz, T. A., and Bame, S. J.: Structural and dynamical aspects of the distant magnetotail determined from ISEE-3 plasma measurements, Planet. Space Sci., 34, 889-901, 1986.

Kaymaz, Z., Petschek, H. E., Siscoe, G. L., Frank, L. A., Ackerson, K. L., and Paterson, W. R.: Disturbance propagation times to the far tail, J. Geophys. Res., 100, 23 743-23 748, 1995.

Powell, K. G., Roe, P. L., Linde, T. J., Gombosi, T. I., and DeZeeuw, D. L.: A solution-adaptive upwind scheme for ideal magnetohydrodynamics, J. Comput. Phys., 154, 284-309, 1999.

Raeder, J.: Global Magnetohydrodynamics - A Tutorial, in: Space Plasma Simulation, edited by: Buechner, J., Dum, C. T., and Scholer, M., Lecture Notes in Physics, vol. 615, Springer Verlag, Heidelberg, 2003.

Toth, G., Sokolov, I. V., Gombosi, T. I., Chesney, D. R., Clauer, C. R., et al.: Space Weather Modeling Framework: A new tool for the space science community, J. Geophys. Res., 110, A12226, doi:10.1029/JA011126, 2005.

Tsyganenko, N. A. and Fairfield, D. H.: Global shape of the magnetotail current sheet as derived from Geotail and Polar data, J. Geophys. Res., 109, A03218, doi:10.1029/2003JA010062, 2004.

Tsyganenko, N. A.: Modeling of twisted/warped magnetospheric configurations using the general deformation method, J. Geophys. Res., 103, 23 551-23 563, 1998. 